\title{
Participação masculina em modalidades de atividades físicas de um Programa para idosos: um estudo longitudinal
}

\author{
Male participation in types of physical activities \\ of a program for the elderly: a longitudinal study
}

Paulo Adão de Medeiros ${ }^{1}$

Inês Amanda Streit ${ }^{1}$

Paula Fabricio Sandreschi ${ }^{1}$

Artur Rodrigues Fortunato ${ }^{1}$

Giovana Zarpellon Mazo ${ }^{1}$
${ }^{1}$ Centro de Ciências da Saúde e do Esporte, Laboratório de Gerontologia, Universidade do Estado de Santa Catarina. R. Pascoal Simone 358, Coqueiros. 88.080-350 Florianópolis SC Brasil. paulofisiosm@yahoo.com.br
Abstract The article seeks to describe male participation on a physical activity program for the elderly in the south of Brazil over a ten-year period (2003-2013). The data of the study were extracted from a database and assessed using descriptive and inferential statistics and assessment of the thematic content. The first phase of the study was longitudinal (from 2003 to 2013) with 163 elderly participants, while the second phase was cross-sectional for the year of 2013 with 45 elderly individuals. Male participation was, on average, 3.5 times lower than female participation for all years assessed, and the activity most practiced was swimming. In the year 2013, the average age of the elderly men was $68 \pm 7.8$ years. Most of them are married, have tertiary education, good acquisitive power, positive self perception of health, one or more diagnosed diseases, and do not participate in any other physical activity outside the program. According to the elderly, the reasons for participating most cited was the search for quality of life and health and, for continuing to exercise, acquiring the taste for the physical activity. Understanding the profile of these individuals and of the reasons that motivate them are important tools to comprehend the issue and help to define public politics directed at this population group.

Key words Motor activity, Men's health, Elderly individuals
Resumo O objetivo do artigo é descrever a participação masculina em um programa de atividades físicas para idosos do sul do Brasil, no período de dez anos (2003-2013). Os dados do estudo foram extraídos de um banco de dados e analisados por meio da estatística descritiva, inferencial e análise de conteúdo temática. O primeiro momento do estudo foi de cunho longitudinal (de 2003 a 2013) e teve 163 idosos participantes e, no segundo momento, foi transversal, referente ao ano de 2013, e contou com 45 idosos. A participação masculina foi, em média, 3,5 vezes menor do que feminina em todos os anos analisados, e a modalidade mais praticada foi a natação. No ano de 2013, a média de idade dos homens idosos foi de $68 \pm 7,8$ anos. $A$ maioria deles é casado, possui elevada escolaridade, bom poder aquisitivo, autopercepção de saúde positiva, possui uma ou mais doenças diagnosticadase não faz outra atividade física fora do programa. De acordo com os idosos, os motivos de ingresso mais citados foram a busca por qualidade de vida e saúde e, de permanência, a aquisição do gosto pela prática de atividade física. A compreensão do perfil desses indivíduos e dos motivos que os sensibilizaram são ferramentas importantes para decifrar essa questão e auxiliar na redefinição de políticas públicas voltadas a essa população.

Palavras-chave Atividade motora, Saúde do homem, Idoso 


\section{Introdução}

O envelhecimento populacional, realidade em quase todas as sociedades, ocorre em diferentes proporções pelo mundo, variando de acordo com as situações econômicas, sociais e políticas de cada país ${ }^{1}$. Em relação aos gêneros, a população masculina vive menos e com inferior qualidade, se comparada com a feminina; segundo o Instituto Brasileiro de Geografia e Estatística (IBGE), a esperança de vida das mulheres ao nascer é de 77 anos, enquanto a dos homens é de 69 anos. A sobremortalidade masculina acontece pelos altos índices de mortes que ocorrem no segmento populacional em plena fase produtiva (dos 25 aos 40 anos de idade $)^{2}$ e pela falta de procura, como fazem as mulheres, aos serviços de atenção primária à saúde e, por isso, adentram ao sistema de saúde para atendimentos já de média e alta complexidade ${ }^{3}$.

A preocupação com a pessoa que envelhece se evidencia por meio de políticas públicas, como a Política Nacional do Idoso ${ }^{4}$, Estatuto do Idoso ${ }^{5} \mathrm{e}$ Política Nacional da Pessoa Idosa ${ }^{6}$. Uma das obrigações das ações governamentais previstas é incentivar e criar projetos de lazer, esporte e atividades físicas que proporcionem a melhoria da qualidade de vida do idoso e estimulem sua participação na comunidade. Além disso, busca articular e ampliar ações para a difusão da atividade física, combate ao sedentarismo e adoção de um estilo de vida mais saudável. Ainda, o envelhecimento ativo, lançado pela Organização Mundial de Saúde (OMS) como uma política de saúde, tem entre um dos seus fatores determinantes a participação em atividades físicas regulares?.

Apesar de ser um direito e uma recomendação ao envelhecimento saudável, a experiência cotidiana e vários estudos sobre a temática apontam que a participação masculina em programas de atividades físicas para a terceira idade é expressivamente inferior à feminina e justificam que isso ocorre, possivelmente, pelo fato dos homens considerarem os projetos existentes inadequados, por não exigirem esforço exaustivo ou por proporem atividades que consideram interferir em sua masculinidade ${ }^{8-10}$.

Nessa mesma perspectiva, surge a Política Nacional de Atenção Integral à Saúde do Homem (PNAISH), que tem por objetivo reduzir a morbimortalidade dos homens brasileiros por meio da ampliação e facilitação do acesso a ações de promoção, prevenção e assistência ${ }^{3}$. No entanto, esse texto não distingue as necessidades peculiares aos homens com mais de 60 anos, indicando que as citadas políticas de saúde ressaltam a descaracterização do idoso sob a perspectiva de gênero ${ }^{11}$.

Nesse contexto, ao reconhecer que os agravos típicos do sexo masculino constituem verdadeiros problemas de saúde pública, infere-se que o menor autocuidado do homem na vida adulta permanece na velhice e pode ser evidenciado por meio de uma menor participação em modalidades de atividades físicas. Acredita-se que a compreensão desse fenômeno, por meio de um olhar sobre a relação que os homens estabelecem com a atividade física na velhice, torna-se importante para a proposição estratégica de medidas e linhas de ação com foco nas características próprias do sexo masculino que venham a promover o acesso dessa população a tal medida de prevenção e promoção da saúde. Assim, o objetivo desse estudo foi descrever a participação masculina em um programa de atividades físicas para idosos do sul do Brasil no período de dez anos (2003-2013).

\section{Material e método}

Essa pesquisa contemplou dois momentos: o primeiro longitudinal, que buscou descrever a participação de idosos do sexo masculino e as modalidades de atividades físicas mais praticadas em dez anos (o período de 2003 a 2013), oferecidas pelo Programa de Extensão Grupo de Estudos da Terceira Idade - GETI, da Universidade do Estado de Santa Catarina - UDESC. O segundo momento foi do tipo transversal, referente ao ano de 2013, e buscou descrever as características sociodemográficas, as condições de saúde, modalidades que participa, atividades físicas praticadas em outros locais e os motivos de ingresso e permanência no Programa.

O GETI/UDESC foi criado em 1989 como um projeto pioneiro na cidade de Florianópolis-SC/ Brasil e atende aproximadamente 350 idosos da comunidade. Ele apresenta projetos de cunho educativo, artístico, cultural e de atividade física. As modalidades de atividades físicas oferecidas atualmente são: caminhada, dança, ginástica, hidroginástica, musculação, natação, pilates e yoga. As aulas destes projetos tem duração de 50 minutos e são realizadas de duas a três vezes por semana, com intensidade moderada ${ }^{12}$. Os critérios de inclusão do estudo foram: idade mínima de 60 anos, ser do sexo masculino e ter se matriculado pelo menos uma vez nos projetos de atividade física do Programa.

O GETI/UDESC ocorre na cidade Florianópolis, SC, que foi considerada em 2013 a capital 
brasileira com melhor Índice de Desenvolvimento Humano $(0,875)^{13}$ e de acordo com o censo de 2010, conta com uma população de 421.240 pessoas, sendo que 48.423 são idosos, 20.529 homens e 27.894 mulheres ${ }^{3}$.

A fonte de coleta de dados foi o banco de dados do Grupo de Pesquisa Atividade Física, Saúde e Envelhecimento - LAGER, reconhecido e certificado pela UDESC e pelo Conselho Nacional de Desenvolvimento Científico - CNPQ, e vinculado ao Programa de Extensão Grupo de Estudos da Terceira Idade - GETI da UDESC. As coletas que originaram o banco de dados foram conduzidas dentro dos padrões exigidos pela resolução n. ${ }^{\circ}$ 196/96 ${ }^{14}$ do Conselho Nacional de Saúde do Brasil. Do banco de dados foram analisados os dados sociodemográficos (idade, estado civil, renda e escolaridade), condições de saúde (auto percepção do estado de saúde, percepção de dificuldade durante a prática de atividade física, doenças e medicamentos), modalidades que participa, prática de atividade física fora do Programa e os motivos de ingresso e permanência.

As análises dos dados quantitativos foram realizadas por meio da estatística descritiva (frequência simples, frequência relativa, média e desvio padrão) e inferencial por meio do pacote de software estatístico MedCalc ${ }^{\circledR}$ (diferença das proporções de homens e mulheres nos dez anos e diferença das proporções da participação masculina no decorrer dos anos). Já os motivos de ingresso e permanência passaram pela análise de conteúdo temática que é um conjunto de técnicas de análise das comunicações visando obter, por procedimentos sistemáticos e objetivos de descrição do conteúdo das mensagens, indicadores que permitam a inferência de conhecimentos relativos às condições de produção/recepção (variáveis inferidas) dessas mensagens ${ }^{15}$.

\section{Resultados}

Inicialmente serão apresentados os resultados da análise longitudinal, na qual verifica-se no Gráfico 1 que durante o período de dez anos, 163 homens idosos foram identificados nas modalidades de atividades físicas (AF). Essa participação foi expressivamente inferior à feminina em todos os anos analisados, sendo em média 3,5 vezes menor e demonstrando significativas proporções de diferença $(\mathrm{p} \leq 0,01)$. Observa-se também que a participação masculina variou de $15 \%$ a $29 \%$ ao longo do período, porém essa variação não apresentou diferença de proporções significativas e o ano com maior participação, tanto relativa quanto absoluta, foi o de 2013.

As modalidades mais praticadas pelos idosos homens no período de dez anos (2003 a 2013) foram natação (24\%) e hidroginástica (15\%), considerando que cada idoso poderia se matricular em mais de uma modalidade no mesmo ano. As demais modalidades oferecidas tiveram baixa participação masculina, conforme pode-se observar no Gráfico 2.

$\mathrm{Na}$ análise transversal, verifica-se na Tabela 1 os dados sóciodemográficos, as condições de saúde e a prática de AF dos homens idosos do GETI/UDESC em 2013. Neste ano, 45 idosos estavam matriculados nas modalidades de AF e apresentavam média de idade de $68 \pm 7,8$ anos. A maioria deles era casado (89\%), possuía elevada

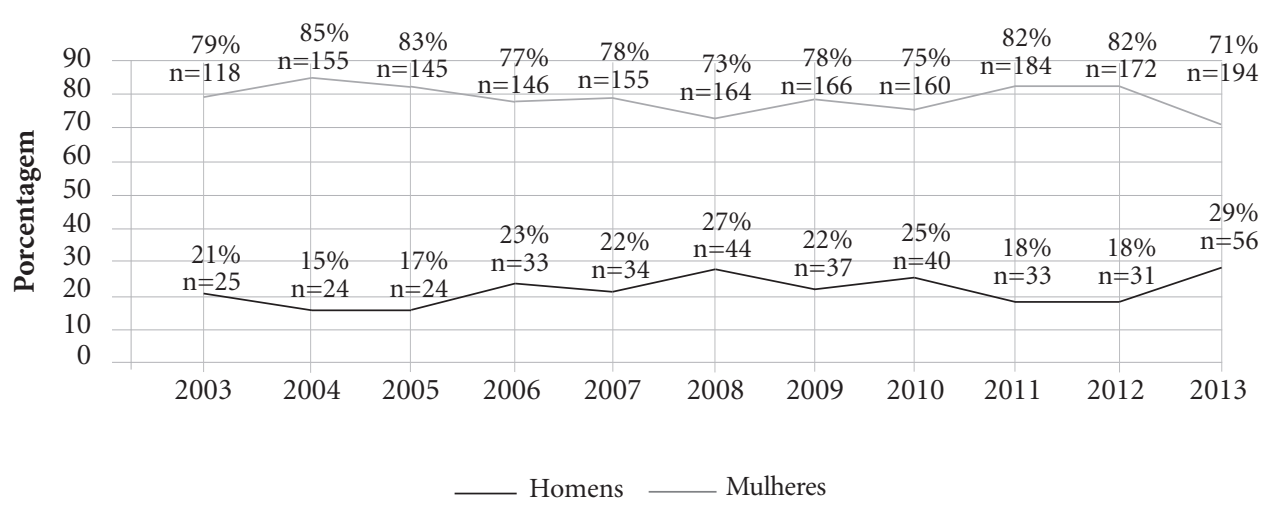

Gráfico 1. Participação de homens e mulheres nos projetos de AF do GETI no período de 2003 a 2013. 


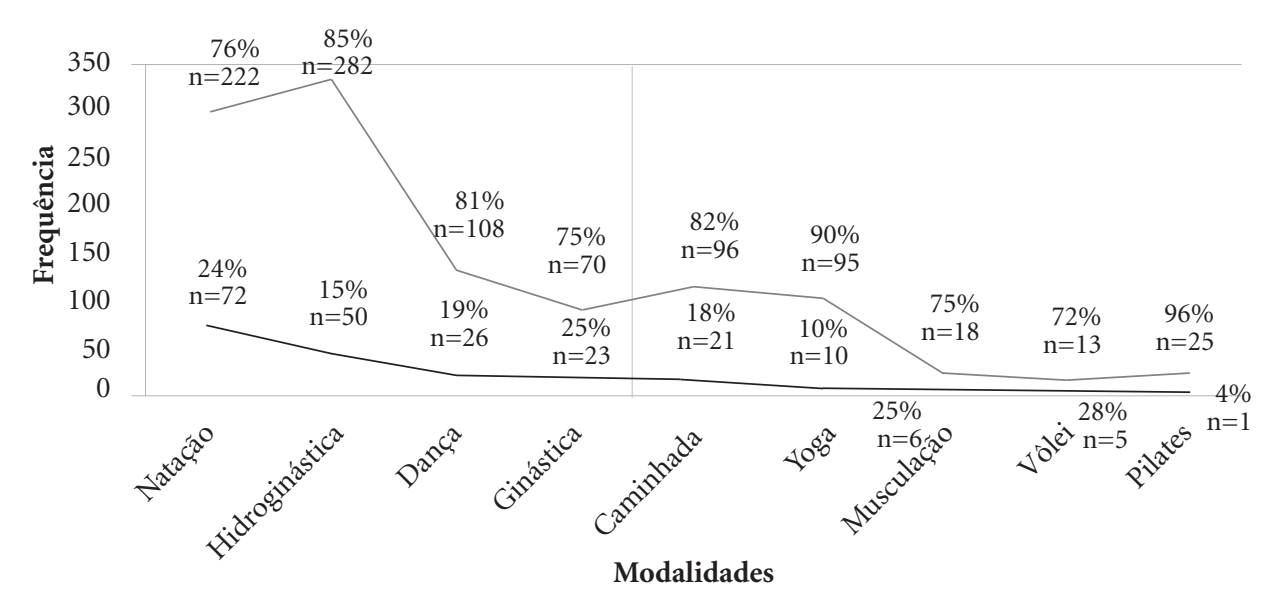

Homens Mulheres

Gráfico 2. Participação de homens e mulheres nas modalidades de AF oferecidas pelo GETI durante os dez anos analisados (2003 a 2013).

escolaridade (82\%), bom poder aquisitivo (56\%), autopercepção positiva do estado de saúde (45\%), possuía uma doença ou mais diagnosticadas (76\%). Ainda, a maioria dos idosos participava da modalidade natação (59\%) e não praticavam regularmente outra atividade física $(80 \%)$, além daquela(s) que realiza $(m)$ no programa.

Os idosos matriculados em 2013 participam em média de 2,5 $\pm 2,2$ anos das modalidades, sendo que três estão há oito anos, identificados como maior tempo de permanência. Verifica-se na Figura 1, que dentre os motivos de ingresso dos idosos nas modalidades de atividade físicas do GETI o mais citado foi a busca por qualidade de vida e a saúde (47\%) o de permanência, o gosto pela prática de atividade física $(29 \%)$. Alguns idosos $(\mathrm{n}=12)$ não relataram o motivo de permanência por terem ingressado no Programa apenas no ano de 2013.

\section{Discussão}

No presente estudo constatou-se que a participação masculina no Programa foi expressivamente inferior à feminina em todos os anos analisados. Resultados semelhantes foram encontrados em estudos transversais realizados com participantes de programas de atividade física orientada para a terceira idade ${ }^{16-18}$.

Benedetti et al. ${ }^{19}$ identificaram inferior participação masculina em grupos de convivência de Florianópolis-SC, de apenas 9,2\%. Também, Borges et al. ${ }^{20}$ identificou uma pequena prevalência de $13,71 \%$ de idosos do sexo masculino em Belo Horizonte (MG). Tratando-se de grupos de atividades físicas para a terceira idade, Valério ${ }^{21}$, em Santa Maria (RS), também constatou pouca adesão masculina, explicando que muitos homens não vão aos grupos por preconceito. Segundo a autora, apesar de estarem conscientes dos benefícios que a atividade física proporciona, os idosos acreditam que já trabalharam muito e que agora está na hora de descansar, livres de compromissos.

Andreotti e Okuma ${ }^{10}$ explicam que é possível que homens acima de 60 anos de idade encontrem mais oportunidades de sociabilização do que as mulheres, citando como exemplo os clubes, praças e bares e, por isso, não procuram os programas de atividade física. Nesta perspectiva, Coutinho e Acosta ${ }^{22} \mathrm{em}$ pesquisa etnográfica sobre ambientes masculinos de lazer, observaram que a baixa participação dos homens em grupos de atividade física acontece por não serem atividades de seu interesse, por considerarem pouco estimulantes e por não sentirem a necessidade de realizar exercícios físicos para manterem seu desempenho.

$\mathrm{Na}$ velhice, o modelo de masculinidade que valoriza o controle das emoções, o machismo e a competitividade tendem a se exacerbar, visto que o idoso vai perdendo suas funções de mando e assim vai sendo relegado a um posto secundário. Essa sensação costuma fazê-lo sentir-se inútil, incapaz e humilhado em decorrência do afastamento do 
Tabela 1. Dados sociodemográficos, condições de saúde e prática de atividade física dos idosos participantes das modalidades de atividades físicas do GETI/UDESC (ano 2013).

\begin{tabular}{|c|c|c|}
\hline \multicolumn{3}{|l|}{ Dados sociodemográficos } \\
\hline \multirow[t]{3}{*}{ Idade } & Média & $\pm \mathrm{DP}$ \\
\hline & 68 & $\pm 7,8$ \\
\hline & $(\mathbf{n})$ & $(\%)$ \\
\hline \multicolumn{3}{|l|}{ Estado civil } \\
\hline Casado & 40 & 89 \\
\hline Separado & 4 & 9 \\
\hline Viúvo & 1 & 2 \\
\hline \multicolumn{3}{|l|}{ Renda mensal } \\
\hline Até três salários mínimos & 7 & 16 \\
\hline De três a cinco salários mínimos & 13 & 29 \\
\hline Mais que seis salários mínimos & 25 & 56 \\
\hline \multicolumn{3}{|l|}{ Escolaridade } \\
\hline Fundamental incompleto & 3 & 7 \\
\hline Fundamental completo & 3 & 7 \\
\hline Médio incompleto & 2 & 4 \\
\hline Médio completo & 19 & 42 \\
\hline Superior completo & 14 & 31 \\
\hline Pós-graduação & 4 & 9 \\
\hline \multicolumn{3}{|l|}{ Condições de saúde } \\
\hline \multicolumn{3}{|l|}{ Autopercepção do estado de saúde } \\
\hline Ótima & 14 & 31 \\
\hline Boa & 20 & 45 \\
\hline Regular & 10 & 22 \\
\hline Ruim & 1 & 2 \\
\hline \multicolumn{3}{|l|}{$\begin{array}{l}\text { Percepção de dificuldade durante a } \\
\text { prática de AF }\end{array}$} \\
\hline Sim & 5 & 11 \\
\hline Não & 40 & 89 \\
\hline \multicolumn{3}{|l|}{ Presença de doença(s) } \\
\hline Sim & 39 & 80 \\
\hline Não & 9 & 20 \\
\hline \multicolumn{3}{|l|}{ Uso de medicamentos } \\
\hline Sim & 34 & 76 \\
\hline Não & 11 & 24 \\
\hline \multicolumn{3}{|l|}{ Prática de AF } \\
\hline \multicolumn{3}{|l|}{ Modalidades praticadas ${ }^{*}$} \\
\hline Natação & 27 & 58,7 \\
\hline Caminhada & 7 & 15,2 \\
\hline Hidroginástica & 6 & 12,9 \\
\hline Dança & 5 & 11,1 \\
\hline Ginástica & 4 & 8,9 \\
\hline Musculação & 3 & 6,7 \\
\hline Pilates & 1 & 2,2 \\
\hline \multicolumn{3}{|l|}{ Prática de outra AF fora do GETI } \\
\hline Sim & 9 & 20 \\
\hline Não & 36 & 80 \\
\hline
\end{tabular}

$\pm \mathrm{DP}=$ desvio padrão; $\mathrm{n}=$ frequência absoluta; $\%=$ frequência relativa; $\mathrm{AF}=$ atividade física. ${ }^{*}=$ os idosos poderiam praticar mais de uma modalidade no mesmo ano.

trabalho, revezes econômicos, problemas de relacionamento ou de desempenho sexual. Ainda, a aposentadoria acarreta numa mudança radical de organização do tempo, dos recursos financeiros e traz uma sensação de desvalorização e ausência de lugar social ${ }^{23}$.

Ribeiro et al. ${ }^{24}$ constataram um perfil diferente de estilo de vida ativo entre os gêneros, com atividades físicas sendo mais frequentemente realizadas pelos homens e as atividades ocupacionais (instrumentais, sociais e intelectuais) mais comuns entre as mulheres. Esse resultado contrapõe os achados do presente estudo, porém a amostra era composta de idosos que realizam atividades fora de um programa sistematizado de atividades físicas.

Tanto a Sociedade Brasileira de Medicina do Esporte (SBME) e a Sociedade Brasileira de Geriatria e Gerontologia (SBGG) concordam que a atividade física regular melhora a qualidade e expectativa de vida do idoso. Além disso, propõe que o governo, demais instituições médicas e científicas e a mídia devem estimular e desenvolver ações para viabilizar e orientar tal prática nessa faixa etária ${ }^{25}$.

Ao refletir sobre os resultados, acredita-se que da mesma maneira que os homens não costumam acessar os serviços de saúde por diversos motivos ligados à construção da masculinidade, estes também durante o seu processo de envelhecimento não buscam participar de projetos sistematizados de atividade física. Alguns dos sentidos atribuídos à masculinidade hegemônica fortalecem a apropriação de representações que associam o masculino à força, à assertividade, à não-vulnerabilidade e a crença de imunidade que internalizadas pelos homens cerceiam a busca pelos serviços de saúde. Esta procura associada ao fato de cuidar de si seriam comportamentos naturalizados como próprios do feminino e também demostrariam uma fragilidade masculina ${ }^{26-29}$. Assim, entende-se que a mesma percepção de que os serviços de saúde não condizem com o estereótipo da figura masculina, os programas de atividades físicas não se caracterizam como ambientes voltados ao masculino.

Vários estudos abordam a construção de identidades masculinas através de práticas corporais e esportivas sob a perspectiva do gênero ${ }^{30-32}$. Oliveira ${ }^{33}$ destaca a estreita relação de valores ditos masculinos com a prática dos esportes, uma vez que desde cedo os meninos eram estimulados ao exercício e o treinamento como uma maneira de torná-los saudáveis, robustos e possuidores de sapiência e sensatez que são características como parte da formação de um ideal de masculinidade.

Salles-Costa et al. ${ }^{34}$ explicam que homens e mulheres apresentam comportamentos distintos no que se refere à prática de exercícios físicos no lazer. Durante a vida adulta, os homens se 
engajam mais em atividades físicas coletivas e de caráter competitivo, e as mulheres, em atividades individuais, que requerem do corpo menos força física. Essa distinção parece não permanecer com o público idoso, pois segundo as modalidades do programa percebe-se um grande destaque para a natação e a hidroginástica, respectivamente.

Simões et al. ${ }^{35}$ relatam que as modalidades aquáticas possuem grande demanda independente dos gêneros, pois dentre as diversas possibilidades de exercícios físicos disponíveis para os idosos, a hidroginástica e a natação vêm se destacando pela conquista cada vez maior de adeptos. $\mathrm{O}$ ambiente aquático por ser particularmente apropriado para essa faixa etária facilita a locomoção, oferecendo menos impacto aos ossos e às articulações destes indivíduos que, naturalmente, estão mais frágeis em virtude do avanço da idade $\mathrm{i}^{36-38}$.

Oliveira $^{33}$ coloca que a ginástica no passado era realizada aos moldes do treinamento militar e designada aos meninos para desenvolver uma boa forma física e atingir um ideal de masculinidade. Porém, acredita-se que atualmente essa modalidade está mais ligada às características femininas (não são muito atrativas ao público masculino), sendo adaptada às particularidades dos idosos e utilizadas dentro dos projetos de terceira idade, para manutenção das capacidades físicas.

Um estudo realizado com 385 idosos ativos fisicamente de ambos os sexos que analisou a prática de atividade física no lazer constatou que a modalidade mais procurada e praticada pelos homens foi a caminhada, seguida da ginástica ${ }^{39}$. Esse resultado vai de encontro ao presente estudo em que a modalidade mais praticada pelos homens foi a natação, seguida da hidroginástica. Essa contraposição pode ser explicada pelo fato do primeiro estudo não contar com idosos que participassem necessariamente de um programa de atividade física orientada e por isso torna-se uma atividade simples, que não necessita de equipamentos especiais ${ }^{40}$.

Todas as modalidades propostas pelo programa classificam-se como práticas de intensidade leve ou moderada conforme Ainsworth et al. ${ }^{41}$ no entanto, entende-se que cada programa tem suas peculiaridades de gestão e organização. Assim, as modalidades de atividades físicas voltadas ao público idoso possuem características próprias de cada localidade, que acabam por influenciar e até mesmo limitar a escolha por parte do público idoso. Acredita-se que essa variação regional entre os tipos de programas também influencia as características do público participante. Considerando os idosos participantes no ano de 2013, percebe-se um perfil de idosos jovens, casados, com alto nível educacional e boa renda.

Em relação às condições de saúde, os idosos do projeto possuem uma ou mais doenças diagnosticadas, entre as quais destacam-se as crônico-degenerativas mais comuns à população idosa. Nesse sentido, entende-se que estes estão conseguindo realizar as recomendações propostas pelo Ministério para esse tipo de paciente, nos quais modificações de estilo de vida são de fundamental importância no processo terapêutico e na prevenção da hipertensão e diabetes, sendo a prática regular de atividade física considerada uma medida prioritária de prevenção e tratamento ${ }^{6,42,43}$.

Além disso, o grupo pesquisado considera sua saúde boa, seus componentes não possuem dificuldades durante a realização dos exercícios e não praticam regularmente outra atividade física além daquela(s) do programa. Pereira e Okuma ${ }^{44}$ analisaram 54 idosos, 14 homens e 40 mulheres de um projeto de extensão da Universidade de São Paulo. A variação da idade dos homens foi de 60 à 80 anos, sendo $71 \%$ com nível superior, com níveis socioeconômicos A e B, e que apresentavam doenças principalmente do sistema cardiorrespiratório e metabólicas, o que vai ao encontro do perfil encontrado nesse estudo.

Gonzalez e Seidl ${ }^{45}$ ao investigarem percepções de homens idosos quanto às limitações e aos ganhos vinculados ao envelhecimento e identificaram que aqueles com maior poder aquisitivo, histórico de trabalho intelectualizado e nível mais elevado de escolaridade destacaram as consequências das limitações físicas como comprometedoras do desempenho em atividades e exercícios físicos, tais como correr, caminhar e praticar esportes, avaliados como importantes para o bem-estar. Por outro lado, os idosos de menor poder aquisitivo e com baixa escolaridade pareceram ressentir-se mais da diminuição na capacidade laborativa. Tal constatação vai ao encontro do perfil do público pesquisado, visto suas características e a importância que atribuem à atividade física.

Segundo dados do Vigitel ${ }^{46}$ a cidade de Florianópolis foi a que apresentou maiores índices para a frequência de adultos que praticam o volume recomendado de atividade física no tempo livre tanto entre os homens (53\%) quanto entre as mulheres $(30,9 \%)$. O percentual de adultos que praticam o volume recomendado de atividade física no tempo livre tendeu a diminuir com o aumento da idade entre os homens, sem que nenhuma relação entre essas variáveis tenha sido encontrada para as mulheres. Em ambos os sexos, a prática do volume recomendado de atividade física no tempo livre 
cresceu com o nível de escolaridade. Ainda, entre homens, a frequência de inatividade física tendeu a aumentar com a idade. No entanto, Cardoso et al. ${ }^{47}$ ao pesquisarem possíveis diferenças entre os gêneros no nível de atividade física de 225 do sexo feminino e 37 do sexo masculino de 37 grupos de convivência de São José - SC identificaram que as mulheres idosas são mais ativas que os homens idosos, porém relatam que essa diferença ocorreu em virtude da sua maior participação nas atividades domésticas.

Spirduso $^{48}$ compreende que, mesmo as pessoas sabendo dos benefícios das atividades físicas e da necessidade de alterar hábitos de vida para a promoção da saúde, muitas não mudariam ou não poderiam modificar suas atitudes. Em relação aos motivos que levaram os homens idosos a ingressar no programa de atividades físicas encontra-se como mais citado a busca pela qualidade de vida e saúde. E como motivo de permanência foi encontrado a aquisição do gosto pela prática de atividade física. Nesse sentido, estudo de Corrêa et $a{ }^{49}$ fornece evidências de que a prática de atividades físicas no lazer e no deslocamento é capaz de diminuir a presença dos sintomas psicológicos e somáticos negativos ligados ao processo de envelhecimento masculino.

Poucos estudos referem os motivos de ingresso e permanência fazendo uma análise em separado dos idosos somente do sexo masculino.

Valério ${ }^{20}$ destaca que apesar do número reduzido de participantes masculinos nos grupos de terceira idade, aqueles que participam vão com o propósito de encontrar uma vivência do envelhecimento mais saudável, superar a solidão e apatia. Assim, identificou como motivos de adesão aos projetos a busca por cuidados à saúde, melhora da autoestima, bem-estar e disposição para as atividades, como também a ocupação do tempo livre que possibilita novas amizades.

Além de sensibilizar os idosos para iniciar a prática de atividade física, a manutenção desta é essencial para a obtenção de benefícios. Meurer et al. ${ }^{50}$ acrescentam que é importante conhecer os principais fatores que mantêm os idosos em programas de exercícios físicos para que melhorem sua saúde e tenham qualidade de vida.

Grande parte das investigações sobre essa temática realiza as análises sem a distinção entre os gêneros, porém os resultados encontram-se em conformidade com o presente estudo. Pereira e Okuma ${ }^{44}$ ao analisar idosos de um Projeto de extensão verificaram que para a adesão nas atividades físicas os principais itens citados foram melhorar o condicionamento físico, adquirir co- nhecimentos, melhorar saúde, melhorar qualidade de vida, aspectos psicológicos, interação social e ocupar o tempo livre. Castro et al. ${ }^{51}$ identificaram que, entre os homens idosos que participam dos programas de exercícios físicos oferecidos pelo SESC do Distrito Federal, os principais motivos de permanência foram o bem-estar pessoal (90,5\%), condicionamento físico $(80,3 \%)$ e prevenção de doenças $(77,5 \%)$.

Nesse sentido, a compreensão dos motivos que levam os idosos a estarem vinculados à atividade física é importante, visto que muitas vezes suas necessidades não vêm sendo consideradas por quem promove as atividades. Assim, são utilizadas estratégias de intervenção cujo estímulo é externo, numa tentativa de levá-lo a alcançar aquilo que é considerado bom pela ciência e não necessariamente pelo próprio sujeito ${ }^{52}$.

\section{Considerações finais}

Os resultados apresentados reafirmam a menor participação masculina em projetos sistematizados de atividade física e acrescentam que esse fenômeno permanece ao longo dos anos. A partir disso, entende-se que as construções culturais da masculinidade afetam o imaginário dos homens durante toda a vida, e inclusive durante a velhice interferem no seu comportamento e na escolha de atividades que irão preencher o seu tempo livre. A crença de imunidade continua gerando um déficit de autocuidado que reflete na menor busca pelos serviços e projetos de saúde, entre os quais aqueles voltados a promoção da saúde e atividade física. Assim, nota-se que o ambiente dos programas de atividade física não se constituem espaços que estão sendo apropriados pelos homens idosos e isso compromete o alcance dos benefícios desta atividade divulgados pelos órgãos governamentais de gestão do setor.

Apesar do grande incentivo e da ampla divulgação dos benefícios da atividade física por diversas políticas governamentais, não é possível observar a sua prática regular em programas estruturados para a população idosa. No entanto, esses sujeitos podem preferir outros tipos de atividades realizadas de maneira individual ou esporádica que não requeiram maiores vínculos ou compromissos.

A noção de invulnerabilidade masculina, muitas vezes, fica comprometida durante o processo de envelhecimento através das perdas de papéis sociais e capacidades físicas que levam os homens a se fecharem ou buscarem estratégias de saúde, como 


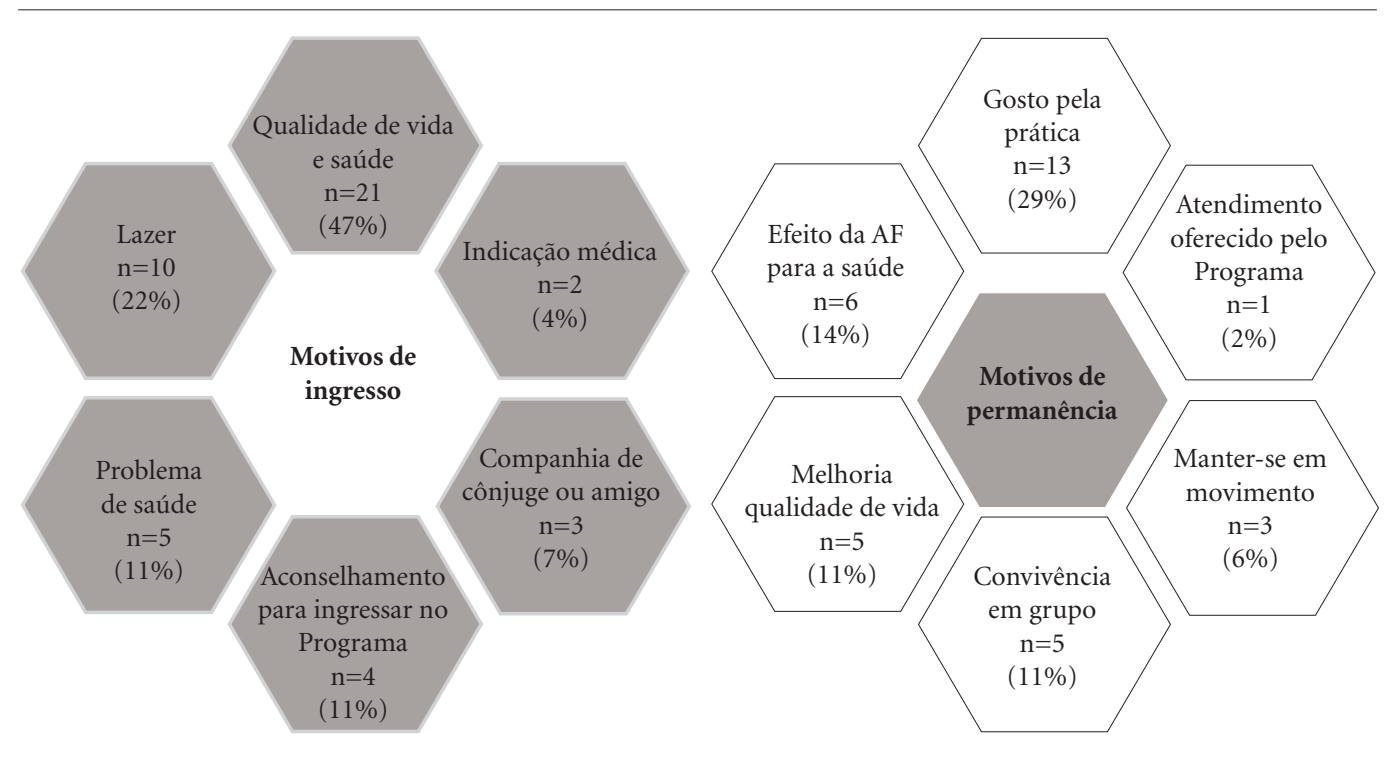

Figura 1. Motivos de ingresso e permanência dos idosos participantes nas modalidades de atividades físicas no GETI/UDESC (ano 2013).

no caso a prática de exercícios físicos. Essa situação sugere que aqueles idosos vinculados aos projetos já estão sensibilizados em maior ou menor grau ao desenvolvimento de hábitos mais saudáveis de vida. A compreensão do perfil desses indivíduos e dos motivos que os sensibilizaram são ferramentas importantes para decifrar essa questão e auxiliar na redefinição de políticas públicas voltadas a essa população. Portanto, sugere-se o desenvolvimento de novos estudos utilizando métodos qualitativos que possam escutar as percepções dos idosos aderentes a estrutura de projetos sistematizados de atividade física.

Sendo os homens idosos um grupo que apresenta vulnerabilidades, sugere-se que o mesmo seja tratado com a devida relevância que merece dentro do sistema de saúde publica. Assim, deve-se considerar a distinção entre os gêneros em relação aos diversos padrões de envelhecimento. O homem apresenta características peculiares e enfrenta situações diferentes ao envelhecer que merecem uma atenção especial e devem ser consideradas ao se propor qualquer tipo de ação voltada a essa população. Nessa perspectiva, é fundamental compreender o sentido atribuído à atividade física pelo homem idoso, além de reflexões sobre questões de gênero, organização e planejamento dos projetos que devem se preparar para acolher as demandas de ambos os sexos de maneira equânime.

\section{Colaboradores}

PA Medeiros trabalhou na concepção e na redação, IA Streit na redação, PF Sandreschi na pesquisa e na metodologia e AR Fortunato na pesquisa e metodologia. GZ Mazo orientou esse estudo.

\section{Agradecimentos}

Ao Conselho Nacional de Desenvolvimento Científico e Tecnológico - CNPq pela bolsa de produtividade em pesquisa destinada à orientadora deste estudo e à Coordenação de Aperfeiçoamento de Pessoal de Nível Superior - CAPES pela bolsa de mestrado concedida ao autor Paulo Adão de Medeiros. 


\section{Referências}

1. Brasil. Ministério da Saúde (MS).Envelhecimento e saúde da pessoa idosa. Brasília: MS; 2007.

2. Instituto Brasileiro de Geografia e Estatística (IBGE). Sintese de indicadores sociais: uma análise das condições de vida da população brasileira - 2010. Rio de Janeiro: IBGE; 2010.

3. Brasil. Ministério da Saúde (MS). Política nacional de atenção integral à saúde do homem: princípios e diretrizes. Brasília: MS; 2009.

4. Brasil. Lei no. 8.842, de 4 de janeiro de 1994. Dispõe sobre a política nacional do idoso, cria o conselho nacional do idoso e dá outras providências. Diário Oficial da União 1994; 4 jan.

5. Brasil. Lei no 10.741 , de 01 de outubro de 2003. Dispõe sobre o estatuto do idoso e dá outras providências. Diário Oficial da União 2003: 1 out.

6. Brasil. Portaria no 2.528 , de 19 de outubro de 2006 . Aprova a Política Nacional de Saúde da Pessoa Idosa. Diário Oficial da União 2006: 19 out.

7. Organização Mundial da Saúde (OMS). Envelhecimento Ativo: uma política de saúde. Brasília: Organização PanAmericana da Saúde; 2005.

8. Ribeiro JAB, Cavalli AS, Cavalli MO, Pogorzelskil LV, Pretes MR, Ricardo LIC. Adesão de idosos a projetos de atividade física: motivação e significância. Rev Bras Ciênc Esporte 2012; 34(4):969-984.

9. Mazo GZ, Cardoso FL, Aguiar DL. Projeto de hidroginástica para idosos: motivação, auto-estima e autoimagem. Rev Bras Cineantropom Desempenho Hum 2006; 8(2):67-72

10. Andreotti M, Okuma S. Perfil sócio-demográfico e de adesão inicial de idosos ingressantes em um projeto de Educação Física. Rev Bras Educ Fís Esporte 2003; 17(2):142-153.

11. Minayo MCS, Meneghel SN, Cavalcante FG. Suicídio de homens idosos no Brasil. Cien Saude Colet 2012; 17(10):2665-2674.

12. Mazo GZ, Cardoso AS, Dias RG, Balbé GP, Virtuoso JF. Do diagnóstico à ação: grupo de estudos da terceira idade: alternativas para a promoção do envelhecimento ativo. Rev Bras Ativ Fís Saúde 2009; 14(1):65-70.

13. Instituto Brasileiro de Geografia e Estatística. Primeiros resultados do Censo demográfico 2010. Rev. Bras. Est. Pop. 2011; 28(1):3-4.

14. Brasil. Ministério da Saúde (MS). Conselho Nacional de Saúde. Resolução nº. 196 de 10 de outubro de 1996. Diretrizes e Normas Regulamentadoras de Pesquisas Envolvendo Seres Humanos. Diário Oficial da União 1996; 16 out.

15. Minayo MCS. O desafio do conhecimento: pesquisa qualitativa em saúde. $11^{\text {a }}$ ed. São Paulo: Hucitec; 2008.

16. Soares ES. A cadeira de balança está vazia: os papéis sociais dos idosos participantes de grupos de convivência na cidade de Fortaleza em Políticas e Sociedade da Universidade Estadual do Ceará. Fortaleza: Universidade Estadual do Ceará; 2010.

17. Yassuda MS, Silva HS. Participação em programas para a terceira idade: impacto sobre a cognição, humor e satisfação com a vida. Estudos de Psicologia 2010; 27(2):207-2014
18. Lima CRV. Políticas públicas para idosos: a realidade das Instituições de Longa Permanência para idosos do Distrito Federal [monografia]. São Paulo: Universidade de São Paulo; 2011.

19. Benedetti TRB, Mazo GZ, Borges LJ. Condições de saúde e nível de atividade física em idosos participantes e não participantes de grupos de convivência de Florianópolis. Cien Saude Colet 2012; 17(8):2087-2093.

20. Borges PL, Bretas RP, Azevedo SF, Barbosa JMM. Perfil dos idosos freqüentadores de grupos de convivência em Belo Horizonte, Minas Gerais, Brasil. Cad Saude Publica 2008; 24(12):2798-2808.

21. Valerio MP. A pouca adesão masculina aos grupos de atividade física para a terceira idade [dissertação]. Santa Maria: Universidade Federal de Santa Maria; 2001.

22. Coutinho RX, Acosta MAF. Ambientes masculinos da terceira idade. Cien Saude Colet 2009; 14(4):1111-1118.

23. Fäber S. Envelhecimento e elaboração de perdas. $A$ terceira idade 2012; 23(53):7-17.

24. Ribeiro, PCC, Neri AL, Cupertino APFB, Yassuda MS. Variabilidade no envelhecimento ativo segundo gênero, idade e saúde. Psicologia em Estudo 2009; 14(3):501-509.

25. Nobrega ACL, Freitas EV, Oliveira MAB, Leitão MB, Lazzoli JK, Nahas RM, Baptista CAS, Drummond FA, Rezende L, Pereira J, Pinto M, Radominski RB, Leite N, Thiele ES, Hernandez AJ, Araújo CGS, Teixeira JAC, Carvalho T, Borges SF, Rose EH. Posicionamento oficial da Sociedade Brasileira de Medicina do Esporte e da Sociedade Brasileira de Geriatria e Gerontologia: atividade física e saúde no idoso. Rev Bras Med Esporte 1999; 5(6):207-211.

26. Mota MP. Gênero e sexualidade: fragmentos de identidade masculina nos tempos da Aids. Cad Saude Publica 1998; 14(1):145-155.

27. Villela WV, Doreto DT. Sobre a experiência sexual dos jovens. Cad Saude Publica 2006; 22(11):2467-2472.

28. Gomes R, Nascimento EF. A produção do conhecimento da saúde pública sobre a relação homem saúde: uma revisão bibliográfica. Cad Saude Publica 2006; 22(5):901-911.

29. Lopez SB, Moreira MCN. Políticas Nacionais de Atenção Integral à Saúde de Adolescentes e Jovens e à Saúde do Homem: interlocuções políticas e masculinidade. Cien Saude Colet 2013; 18(3):743-752.

30. Pereira EGB. Discutindo gênero, corpo e masculinidade. In: Romero E, Pereira EGB, organizadores. Universo do corpo: masculinidades e feminilidades. Rio de Janeiro: Shape; 2008. p. 87-101.

31. Pereira EGB. Reflexões sobre práticas corporais, identidades e masculinidades. Rev Bras Psicol Esporte Motricidade Hum 2009; 1:37-43.

32. Devide FP, Batista RS. O exercício físico na construção da identidade de gênero: por uma masculinidade plural. In: Knijnik JD, organizador. Gênero e esporte: masculinidades e feminilidades. Rio de Janeiro: Apicuri; 2010. p. 185-209.

33. Oliveira PP. A construção social da masculinidade. Belo Horizonte: UFMG; 2004.

34. Salles-Costa R, Heilborn ML, Werneck GL, Faerstein E, Lopes CS. Gênero e prática de atividade física de lazer. Cad Saude Publica 2003: 19(Supl. 2):S325-S333. 
35. Simões RR, Portes Júnior M, Moreira WW. Idosos e hidroginástica: corporeidade e vida. $R$. bras. Ci. e Mov. 2011; 19(4):40-50.

36. Baum G. Aquaeróbica manual de treinamento. São Paulo: Manole; 2000.

37. Aidar FJ, Idar FJ, Silva AJ, Reias VM, Carneiro AL, Leite TM. Idosos e adultos velhos: atividades físicas aquáticas e a autonomia funcional. Fitness \& Performance Journal 2006; 5(5):271-276.

38. Cerri AS, Simões R. Hidroginásticas e idosos: por que eles praticam? Rev Movimento 2007; 13(1):81-92.

39. Salvador EP, Florindo AA, Reis RS, Costa EF. Percepção do ambiente e prática de atividade física no lazer entre idosos. Rev Saude Publica 2009; 43(6):972-980.

40. Nunes MES, Santos S. Avaliação funcional de idosos em três projetos de atividade física: caminhada, hidroginástica e Lian Gong. Rev. Port. Cien. Desp. 2009, 9(2-3):150-159.

41. Ainsworth BE, Haskell WL, Whitt MC, Irwin ML, Swartz AM, Strath SJ, O’Brien WL, Bassett Jr DR, Schmitz KH, Emplaincourt PO, Jacobs Jr DR, Leon AS. Compendium of physical activity codes and MET intensities. Med. Sci. Sports Exerc 2000; 32(Supl. 9):S498-504.

42. Brasil. Ministério da Saúde (MS). Secretaria de Atenção à Saúde. Departamento de Atenção Básica. Diabetes Mellitus. Brasília: MS; 2006. (Cadernos de Atenção Básica, n. 16) (Série A. Normas e Manuais Técnicos).

43. Brasil. Ministério da Saúde (MS). Secretaria de Atenção à Saúde. Departamento de Atenção Básica. Hipertensão arterial sistêmica para o Sistema Único de Saúde. Brasília: MS; 2006. (Cadernos de Atenção Básica; 16) (Série A. Normas e Manuais Técnicos)

44. Pereira JRP, Okuma SS. O perfil dos ingressantes de um projeto de educação física para idosos e os motivos de adesão inicial. Rev. bras. educ. fís. esporte. 2009; 23(4):319-334.
45. Gonzales LMB, Seidl EM. O envelhecimento na perspectiva de homens idosos. Paidéia 2011;21(50):345-352.

46. Brasil. Ministério da Saúde (MS). Secretaria de Vigilância em Saúde. Vigitel Brasil 2011: Vigilância de Fatores de Risco e Proteção para Doenças Crônicas por Inquérito Telefônico. Brasília: MS; 2012. (Série G. Estatística e Informação em Saúde).

47. Cardoso AS, Levandoski G, Mazo GZ, Prado APM, Cardoso LS. Comparação do nível de atividade física em relação ao gênero de idosos participantes de grupos de convivência. RBCEH 2008; 5(1):9-18.

48. Spirduso WW. Envelhecimento - aspectos fisiológicos. São Paulo: Manole; 2005.

49. Corrêa LQ, Rombaldi AJ, Silva MC. Atividade Física e Sintomas do Envelhecimento Masculino em uma População do Sul do Brasil. Rev Bras Med Esporte 2011; 17(3): 228-231.

50. Meurer ST, Benedetti TR, Mazo GZ. Teoria da autodeterminação: compreensão dos fatores motivacionais e autoestima de idosos praticantes de exercícios físicos. R. bras. de Atividade Física e Saúde 2011; 16(1):18-23.

51. Castro MS, Miranda M, Silva NL, Palma A, Resende HG. Motivos de ingresso em programas de exercícios físicos oferecidos pelo Serviço Social do Comércio - SESC-DF. Movimento 2009: 15(2):87-102.

52. Okuma SS. O idoso e a atividade física: fundamentos e pesquisa. Campinas: Papirus; 1998.

Artigo apresentado em 05/07/2013

Aprovado em 25/08/2013

Versão final apresentada em 05/09/2013 